\title{
Another Criterion For The Riemann Hypothesis
}

\author{
Frank Vega
}

CopSonic, 1471 Route de Saint-Nauphary 82000 Montauban, France

\begin{abstract}
Let's define $\delta(x)=\left(\sum_{q \leq x} \frac{1}{q}-\log \log x-B\right)$, where $B \approx 0.2614972128$ is the Meissel-Mertens constant. The Robin theorem states that $\delta(x)$ changes sign infinitely often. Let's also define $S(x)=\theta(x)-x$, where $\theta(x)$ is the Chebyshev function. It is known that $S(x)$ changes sign infinitely often. We define the another function $\varpi(x)=\left(\sum_{q \leq x} \frac{1}{q}-\log \log \theta(x)-B\right)$. We prove that when the inequality $\varpi(x) \leq 0$ is satisfied for some number $x \geq 3$, then the Riemann hypothesis should be false. The Riemann hypothesis is also false when the inequalities $\delta(x) \leq 0$ and $S(x) \geq 0$ are satisfied for some number $x \geq 3$ or when $\frac{3 \times \log x+5}{8 \times \pi \times \sqrt{x}+1.2 \times \log x+2}+\frac{\log x}{\log \theta(x)} \leq 1$ is satisfied for some number $x \geq 13.1$.
\end{abstract}

Keywords: Riemann hypothesis, Nicolas inequality, Chebyshev function, prime numbers 2000 MSC: 11M26, 11A41, 11A25

\section{Introduction}

In mathematics, the Riemann hypothesis is a conjecture that the Riemann zeta function has its zeros only at the negative even integers and complex numbers with real part $\frac{1}{2}$ [1]. Let $N_{n}=$ $2 \times 3 \times 5 \times 7 \times 11 \times \cdots \times p_{n}$ denotes a primorial number of order $n$ such that $p_{n}$ is the $n^{\text {th }}$ prime number. Say Nicolas $\left(p_{n}\right)$ holds provided

$$
\prod_{q \mid N_{n}} \frac{q}{q-1}>e^{\gamma} \times \log \log N_{n} .
$$

The constant $\gamma \approx 0.57721$ is the Euler-Mascheroni constant, log is the natural logarithm, and $q \mid N_{n}$ means the prime number $q$ divides to $N_{n}$. The importance of this property is:

Theorem 1.1. [2]. Nicolas $\left(p_{n}\right)$ holds for all prime numbers $p_{n}>2$ if and only if the Riemann hypothesis is true.

In mathematics, the Chebyshev function $\theta(x)$ is given by

$$
\theta(x)=\sum_{p \leq x} \log p
$$

where $p \leq x$ means all the prime numbers $p$ that are less than or equal to $x$. We know this property for this function:

Email address: vega.frank@gmail.com (Frank Vega)

Preprint submitted to Elsevier

October 13, 2021 
Theorem 1.2. [3].

$$
\lim _{x \rightarrow \infty} \frac{\theta(x)}{x}=1
$$

Let's define $S(x)=\theta(x)-x$. It is a known result that:

Theorem 1.3. [4]. $S(x)$ changes sign infinitely often.

We also know that

Theorem 1.4. [5]. If the Riemann hypothesis holds, then

$$
\left(\frac{e^{-\gamma}}{\log x} \times \prod_{q \leq x} \frac{q}{q-1}-1\right)<\frac{3 \times \log x+5}{8 \times \pi \times \sqrt{x}}
$$

for all numbers $x \geq 13.1$.

Let's define $H=\gamma-B$ such that $B \approx 0.2614972128$ is the Meissel-Mertens constant [6]. We know from the constant $H$, the following formula:

Theorem 1.5. [7].

$$
\sum_{q}\left(\log \left(\frac{q}{q-1}\right)-\frac{1}{q}\right)=\gamma-B=H .
$$

For $x \geq 2$, the function $u(x)$ is defined as follows

$$
u(x)=\sum_{q>x}\left(\log \left(\frac{q}{q-1}\right)-\frac{1}{q}\right) .
$$

Nicolas showed that

Theorem 1.6. [2]. For $x \geq 2$ :

$$
0<u(x) \leq \frac{1}{2 \times(x-1)}
$$

Let's define:

$$
\delta(x)=\left(\sum_{q \leq x} \frac{1}{q}-\log \log x-B\right) .
$$

Robin theorem states the following result:

Theorem 1.7. [8]. $\delta(x)$ changes sign infinitely often.

In addition, the Mertens second theorem states that:

Theorem 1.8. [6].

$$
\lim _{x \rightarrow \infty} \delta(x)=0
$$

Besides, we use the following theorems:

Theorem 1.9. [9]. For $x>-1$ :

$$
\frac{x}{x+1} \leq \underset{2}{\log (1+x)}
$$


Theorem 1.10. [10]. For $x \geq 1$ :

$$
\log \left(1+\frac{1}{x}\right)<\frac{1}{x+0.4}
$$

We define another function:

$$
\varpi(x)=\left(\sum_{q \leq x} \frac{1}{q}-\log \log \theta(x)-B\right) .
$$

Putting all together yields the proof that the inequality $\varpi(x)>u(x)$ is satisfied for a number $x \geq 3$ if and only if Nicolas $(p)$ holds, where $p$ is the greatest prime number such that $p \leq x$. In this way, we introduce another criterion for the Riemann hypothesis based on the Nicolas criterion and deduce some of its consequences.

\section{Results}

Theorem 2.1. The inequality $\varpi(x)>u(x)$ is satisfied for a number $x \geq 3$ if and only if $\operatorname{Nicolas}(p)$ holds, where $p$ is the greatest prime number such that $p \leq x$.

Proof. We start from the inequality:

$$
\varpi(x)>u(x)
$$

which is equivalent to

$$
\left(\sum_{q \leq x} \frac{1}{q}-\log \log \theta(x)-B\right)>\sum_{q>x}\left(\log \left(\frac{q}{q-1}\right)-\frac{1}{q}\right) .
$$

Let's add the following formula to the both sides of the inequality,

$$
\sum_{q \leq x}\left(\log \left(\frac{q}{q-1}\right)-\frac{1}{q}\right)
$$

and due to the theorem 1.5 , we obtain that

$$
\sum_{q \leq x} \log \left(\frac{q}{q-1}\right)-\log \log \theta(x)-B>H
$$

because of

$$
H=\sum_{q \leq x}\left(\log \left(\frac{q}{q-1}\right)-\frac{1}{q}\right)+\sum_{q>x}\left(\log \left(\frac{q}{q-1}\right)-\frac{1}{q}\right)
$$

and

$$
\sum_{q \leq x} \log \left(\frac{q}{q-1}\right)=\sum_{q \leq x} \frac{1}{q}+\sum_{q \leq x}\left(\log \left(\frac{q}{q-1}\right)-\frac{1}{q}\right) .
$$

Let's distribute it and remove $B$ from the both sides:

$$
\sum_{q \leq x} \log \left(\frac{q}{q-1}\right)>\gamma+\log \log \theta(x)
$$


since $H=\gamma-B$. If we apply the exponentiation to the both sides of the inequality, then we have that

$$
\prod_{q \leq x} \frac{q}{q-1}>e^{\gamma} \times \log \theta(x)
$$

which means that Nicolas $(p)$ holds, where $p$ is the greatest prime number such that $p \leq x$. The same happens in the reverse implication.

Theorem 2.2. The Riemann hypothesis is true if and only if the inequality $\varpi(x)>u(x)$ is satisfied for all numbers $x \geq 3$.

Proof. This is a direct consequence of theorems 1.1 and 2.1.

Theorem 2.3. If the inequality $\varpi(x) \leq 0$ is satisfied for some number $x \geq 3$, then the Riemann hypothesis should be false.

Proof. This is an implication of theorems 1.6, 2.1 and 2.2.

Theorem 2.4. If the inequalities $\delta(x) \leq 0$ and $S(x) \geq 0$ are satisfied for some number $x \geq 3$, then the Riemann hypothesis should be false.

Proof. If the inequalities $\delta(x) \leq 0$ and $S(x) \geq 0$ are satisfied for some number $x \geq 3$, then we obtain that $\varpi(x) \leq 0$ is also satisfied, which means that the Riemann hypothesis should be false according to the theorem 2.3 .

\section{Theorem 2.5.}

$$
\lim _{x \rightarrow \infty} \varpi(x)=0 .
$$

Proof. We know that $\lim _{x \rightarrow \infty} \varpi(x)=0$ for the $\operatorname{limits}_{\lim _{x \rightarrow \infty}} \delta(x)=0$ and $\lim _{x \rightarrow \infty} \frac{\theta(x)}{x}=1$. In this way, this is a consequence from the theorems 1.8 and 1.2.

Theorem 2.6. If the Riemann hypothesis holds, then

$$
\frac{3 \times \log x+5}{8 \times \pi \times \sqrt{x}+1.2 \times \log x+2}+\frac{\log x}{\log \theta(x)}>1
$$

for all numbers $x \geq 13.1$.

Proof. Under the assumption that the Riemann hypothesis is true, then we would have

$$
\prod_{q \leq x} \frac{q}{q-1}<e^{\gamma} \times \log x \times\left(1+\frac{3 \times \log x+5}{8 \times \pi \times \sqrt{x}}\right)
$$

after of distributing the terms based on the theorem 1.4 for all numbers $x \geq 13.1$. If we apply the logarithm to the both sides of the previous inequality, then we obtain that

$$
\sum_{q \leq x} \log \left(\frac{q}{q-1}\right)<\gamma+\log \log x+\log \left(1+\frac{3 \times \log x+5}{8 \times \pi \times \sqrt{x}}\right) .
$$

That would be equivalent to

$$
\sum_{q \leq x} \frac{1}{q}+\sum_{q \leq x}\left(\log \left(\frac{q}{q-1}\right)-\frac{1}{q}\right)<\gamma+\log \log x+\frac{3 \times \log x+5}{8 \times \pi \times \sqrt{x}+1.2 \times \log x+2}
$$


where we know that

$$
\begin{aligned}
\log \left(1+\frac{3 \times \log x+5}{8 \times \pi \times \sqrt{x}}\right) & <\frac{1}{\frac{8 \times \pi \times \sqrt{x}}{3 \times \log x+5}+0.4} \\
& =\frac{3 \times \log x+5}{8 \times \pi \times \sqrt{x}+0.4 \times(3 \times \log x+5)} \\
& =\frac{3 \times \log x+5}{8 \times \pi \times \sqrt{x}+1.2 \times \log x+2}
\end{aligned}
$$

according to theorem 1.10 since $\frac{8 \times \pi \times \sqrt{x}}{3 \times \log x+5} \geq 1$ for all numbers $x \geq 13.1$. We use the theorems 1.5 and 1.6 to show that

$$
\sum_{q \leq x}\left(\log \left(\frac{q}{q-1}\right)-\frac{1}{q}\right)=H-u(x)
$$

and $\gamma=H+B$. So,

$$
H-u(x)<H+B+\log \log x-\sum_{q \leq x} \frac{1}{q}+\frac{3 \times \log x+5}{8 \times \pi \times \sqrt{x}+1.2 \times \log x+2}
$$

which is the same as

$$
H-u(x)<H-\delta(x)+\frac{3 \times \log x+5}{8 \times \pi \times \sqrt{x}+1.2 \times \log x+2} .
$$

We eliminate the value of $H$ and thus,

$$
-u(x)<-\delta(x)+\frac{3 \times \log x+5}{8 \times \pi \times \sqrt{x}+1.2 \times \log x+2}
$$

which is equal to

$$
u(x)+\frac{3 \times \log x+5}{8 \times \pi \times \sqrt{x}+1.2 \times \log x+2}>\delta(x) .
$$

We know from the theorem 2.1 that $\varpi(x)>u(x)$ for all numbers $x \geq 13.1$ and therefore,

$$
\varpi(x)+\frac{3 \times \log x+5}{8 \times \pi \times \sqrt{x}+1.2 \times \log x+2}>\delta(x) .
$$

Hence,

$$
\frac{3 \times \log x+5}{8 \times \pi \times \sqrt{x}+1.2 \times \log x+2}>\log \log \theta(x)-\log \log x .
$$

Suppose that $\theta(x)=\epsilon \times x$ for some constant $\epsilon>1$. Then,

$$
\begin{aligned}
\log \log \theta(x)-\log \log x & =\log \log (\epsilon \times x)-\log \log x \\
& =\log (\log x+\log \epsilon)-\log \log x \\
& =\log \left(\log x \times\left(1+\frac{\log \epsilon}{\log x}\right)\right)-\log \log x \\
& =\log \log x+\log \left(1+\frac{\log \epsilon}{\log x}\right)-\log \log x \\
& =\log \left(1+\frac{\log \epsilon}{\log x}\right) .
\end{aligned}
$$


In addition, we know that

$$
\log \left(1+\frac{\log \epsilon}{\log x}\right) \geq \frac{\log \epsilon}{\log \theta(x)}
$$

using the theorem 1.9 since $\frac{\log \epsilon}{\log x}>-1$ when $\epsilon>1$. Certainly, we will have that

$$
\log \left(1+\frac{\log \epsilon}{\log x}\right) \geq \frac{\frac{\log \epsilon}{\log x}}{\frac{\log \epsilon}{\log x}+1}=\frac{\log \epsilon}{\log \epsilon+\log x}=\frac{\log \epsilon}{\log \theta(x)}
$$

Thus,

$$
\frac{3 \times \log x+5}{8 \times \pi \times \sqrt{x}+1.2 \times \log x+2}>\frac{\log \epsilon}{\log \theta(x)} .
$$

If we add the following value of $\frac{\log x}{\log \theta(x)}$ to the both sides of the inequality, then

$$
\frac{3 \times \log x+5}{8 \times \pi \times \sqrt{x}+1.2 \times \log x+2}+\frac{\log x}{\log \theta(x)}>\frac{\log \epsilon}{\log \theta(x)}+\frac{\log x}{\log \theta(x)}=\frac{\log \epsilon+\log x}{\log \theta(x)}=\frac{\log \theta(x)}{\log \theta(x)}=1 .
$$

We know this inequality is satisfied when $0<\epsilon \leq 1$ since we would obtain that $\frac{\log x}{\log \theta(x)} \geq 1$. Therefore, the proof is done.

Theorem 2.7. If the inequality $\frac{3 \times \log x+5}{8 \times \pi \times \sqrt{x}+1.2 \times \log x+2}+\frac{\log x}{\log \theta(x)} \leq 1$ is satisfied for some number $x \geq$ 13.1, then the Riemann hypothesis should be false.

Proof. This is a direct consequence of theorem 2.6.

\section{References}

[1] P. B. Borwein, S. Choi, B. Rooney, A. Weirathmueller, The Riemann Hypothesis: A Resource for the Afficionado and Virtuoso Alike, Vol. 27, Springer Science \& Business Media, 2008.

[2] J.-L. Nicolas, Petites valeurs de la fonction d'Euler, Journal of number theory 17 (3) (1983) $375-388$. doi:10.1016/0022-314X(83)90055-0.

[3] T. H. Grönwall, Some asymptotic expressions in the theory of numbers, Transactions of the American Mathematical Society 14 (1) (1913) 113-122. doi:10.2307/1988773.

[4] D. J. Platt, T. S. Trudgian, On the first sign change of $\theta(x)-x$, Math. Comput. 85 (299) (2016) $1539-1547$. doi: $10.1090 / \mathrm{mcom} / 3021$.

[5] J. B. Rosser, L. Schoenfeld, Sharper Bounds for the Chebyshev Functions $\theta(\mathrm{x})$ and $\psi(\mathrm{x})$, Mathematics of computation (1975) 243-269doi:10.1090/S0025-5718-1975-0457373-7.

[6] F. Mertens, Ein Beitrag zur analytischen Zahlentheorie., J. reine angew. Math. 1874 (78) (1874) 46-62. doi:10.1515/crll.1874.78.46. URL https://doi.org/10.1515/crll.1874.78.46

[7] Y. Choie, N. Lichiardopol, P. Moree, P. Solé, On Robin's criterion for the Riemann hypothesis, Journal de Théorie des Nombres de Bordeaux 19 (2) (2007) 357-372. doi:10.5802/jtnb.591.

[8] G. Robin, Sur l'ordre maximum de la fonction somme des diviseurs, Séminaire Delange-Pisot-Poitou Paris 82 (1981) 233-242.

[9] L. Kozma, Useful Inequalities, http://www.lkozma.net/inequalities_cheat_sheet/ineq.pdf, accessed on 2021-10-13 (2021).

[10] A. Ghosh, An Asymptotic Formula for the Chebyshev Theta Function, arXiv preprint arXiv:1902.09231. 